\title{
ANOMALOUS BRANCHING PATTERN OF ARTERY OF HIND GUT-CT ANGIOGRAPHY BASED STUDY
}

\section{Bali Sharma ${ }^{1}$, Neha Saini ${ }^{*}{ }^{2}$,Dhiraj Saxena ${ }^{3}$.}

${ }^{1}$ Assistant professor, Department of Anatomy, SMBT Medical college, Nashik, Maharashtra, India.

${ }^{* 2}$ Research scholar, Department of Anatomy, S.MS. Medical College Jaipur, Rajasthan, India.

${ }^{3}$ Professor, Department of Anatomy, S.M.S .Medical college Jaipur,Rajasthan, India.

\section{ABSTRACT}

The aim of this study was to notice and describe the subsistence and occurrence of anatomical variations of the inferior mesenteric artery by Using imaging technique.

Materials and Methods: 100 patients visited in the radiology department of S.M.S. Medical College Jaipur. To cover the whole abdominal arteria maxima in each patient, spiral CT angiography scan was made and thin slices $(0.6 \mathrm{~mm})$ axial images was obtained, Both sagittal and coronal images was reconstructed.

Results: We found that $83 \%$ of patients presented a classic anatomy of the inferior mesenteric artery. Using this imaging technique; we found the existence of variations of these abdominal blood vessels in $17 \%$ of patients.

Conclusion: The arterial variations should not be ignored and with an accurate knowledge on the anatomical variations, many operative and post operative complications can be avoided. The knowledge of the inferior mesenteric variations would enable the radiologists in protecting the impor-tant vessels prior to transcatheter therapies, and also in preventing inadvertent injuries.

KEYWORDS: Vessels, Inferior Mesenteric Artery,Angiography, Variations.

Corresponding Author: Dr. Neha Saini, Research Scholar, Department of Anatomy,S.M.S. Medical College Jaipur,Rajasthan, India. E-Mail: neha.saini941@gmail.com

Access this Article online Quick Response code

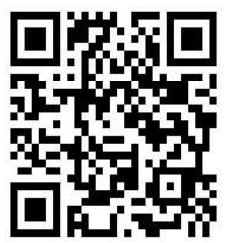

DOI: $10.16965 /$ ijar.2020.174

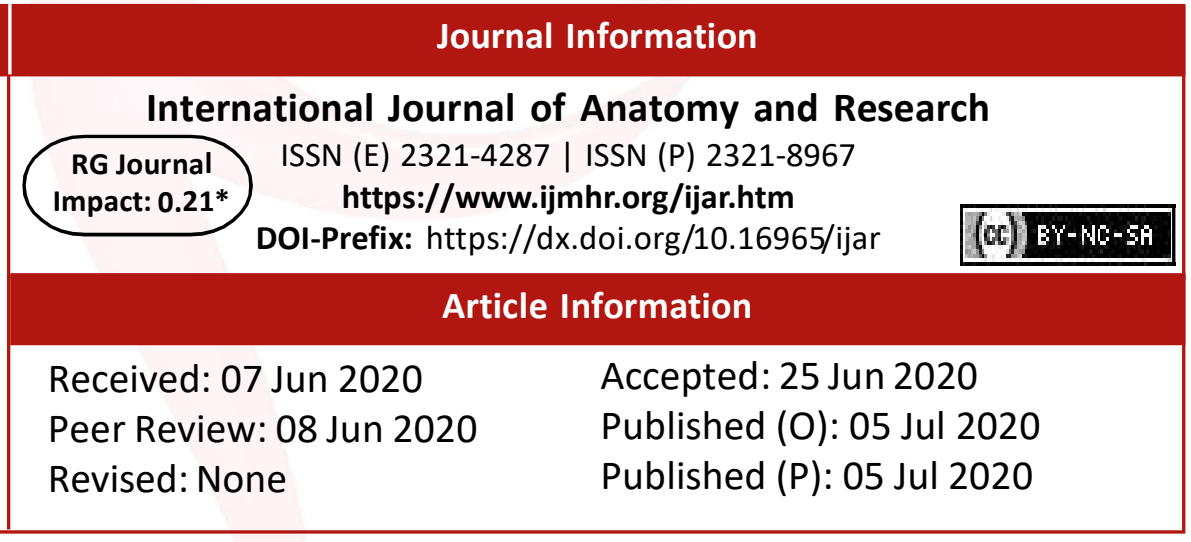

\section{INTRODUCTION}

The vertebral level of emergence of inferior mesenteric artery lies at the level of L3 vertebra from its left anterio-lateral aspect.The origin of the artery is found above the termination of arteria maxima. This then runs in a downward direction below the peritoneum [1].

This artery then navigates through the emergence of left common iliac artery medial to left ureter, and then it again runs in down ward direction in peritoneal fold surrounding sigmoid colon and thus carries on as superior hemorrhoidal artery and provides branches namely, left colic, sigmoidal and superior rectal artery.

Anatomic variations of the gut vessels is fundamental to effectively achieve careful, oncologic, or interventional methodology, aortic supplanting with re-implantation of the artery. All of which can conceivably make noteworthy dismalness as a result of the enormous instinctive region provided by a solitary vessel[2]. Profound information on morphological varieties ventral parts of abdominal arteria maxima is significant for wide spread improvement, different medical procedures 
identified with mesenteric vessels [3]. Present examination incorporates $\mathrm{CT}$-angiography which may help in the field of clinical training and aspects [4].

Aim and objectives: The point of this investigation was to distinguish and portray the presence and occurrence of anatomical varieties of the inferior mesenteric course by utilizing imaging procedure.

\section{MATERIALS AND METHODS}

Study Area:The study was conducted in Department of Anatomy collaboration with Department of Radiology, SMS Medical College \& Hospital, Jaipur (Rajasthan).

Study Sample:CT angiography was randomly selected from the records of 100 patients visited the radiology department of SMS Medical College \& Hospital, Jaipur.

To cover the whole abdominal arteria maxima in each patient, spiral CT angiography scan was made and thin slices $(0.6 \mathrm{~mm})$ axial images were obtained. Both sagittal and coronal images were reconstructed. 3-D reconstruction was done from the data gained by the spiral CT examination. Manipulation of the 3-D images was done by rotation to get the correct planes and deletion of unnecessary anatomical details to clarify the arteries away from superimposed structures. Data was recorded into DVD.

Inclusion Criteria:All subjects undergoing the computed tomographic angiography of the abdominal arteria maxima for any medical or surgical indications were included in study group.

Exclusion Criteria:Subjects who have- Arterial pathology such as aneurysm or tumours, excluded from this study.

\section{RESULTS}

In the clinical trial conducted on total 100 patients from which we found following results. [Table 1\&Graph1]

Type I Normal branching pattern in angiographic study it was in 83 cases i.e. $83 \%$ out of 100 patients. In this pattern inferior mesenteric artery provides left colic, sigmoidal \& superior rectal arteries (fig 1).

Type II Pattern was observed in 6 cases i.e. 6\%, in that case absence of left colic artery; inferior mesenteric artery gives only sigmoidal \& superior rectal arteries (fig.2).

Type III Pattern was observed in 3 cases i.e.3\%, in that case presence of double left colic artery (fig.3),

Type IV Pattern was observed in 4 sufferers i.e. $4 \%$, in that pattern presence of accessory sigmoidal artery from left colic artery (fig. 4).

Type V Pattern of inferior mesenteric artery according angiography was observed in 4 cases i.e. $4 \%$, in that case sigmoidal \& left colic artery arise from common trunk (fig. 5).

Table 1:Branching pattern of inferior mesenteric artery in males and females.

\begin{tabular}{ccccccc}
\hline Branching pattern & Male & \multicolumn{3}{c}{ Female } & \multicolumn{3}{c}{ Total } \\
& No & $\%$ & No & $\%$ & No & $\%$ \\
Type I & 50 & 83.34 & 33 & 82.25 & 83 & $83 \%$ \\
Type II & 5 & 8.34 & 1 & 2.5 & 6 & $6 \%$ \\
Type III & 0 & 0 & 3 & 7.5 & 3 & $3 \%$ \\
Type IV & 3 & 5 & 1 & 2.5 & 4 & $4 \%$ \\
Type V & 2 & 3.34 & 2 & 5 & 4 & $4 \%$ \\
Total & 60 & 100 & 40 & 100 & 100 & 100 \\
\hline
\end{tabular}

\section{Branching pattern of inferior mesenteric artery}

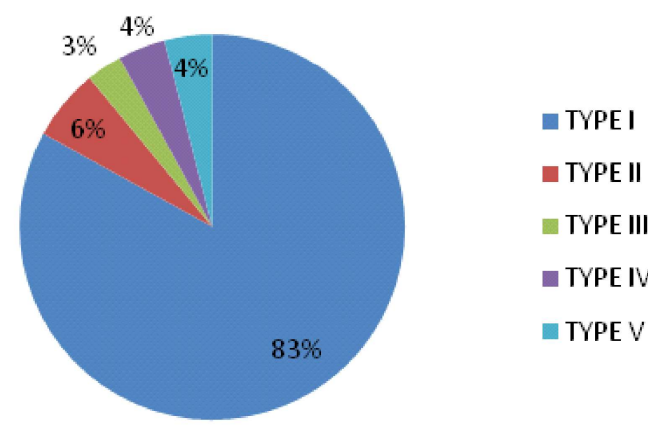

Graph 1: Branching pattern of interior mesenteric artery (angiographic study).

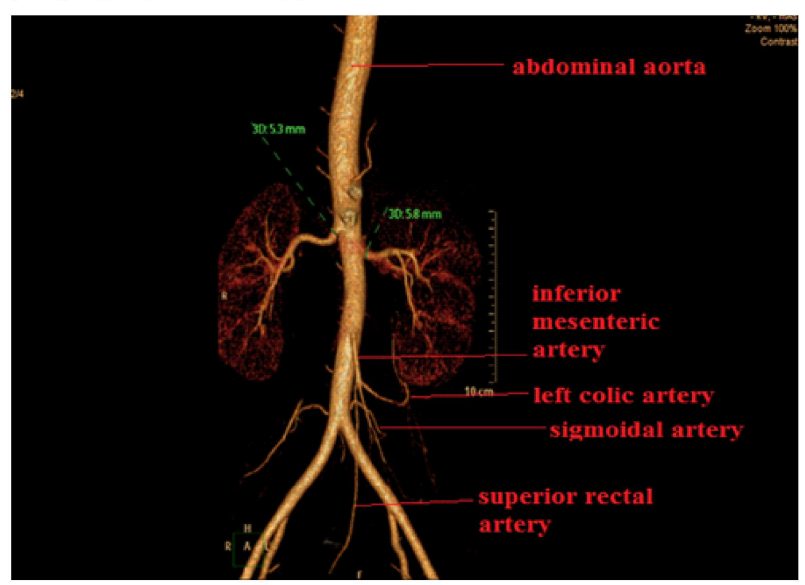

Fig. 1: Type I -Normal Branching Pattern of Inferior Mesenteric Artery (83\%) 


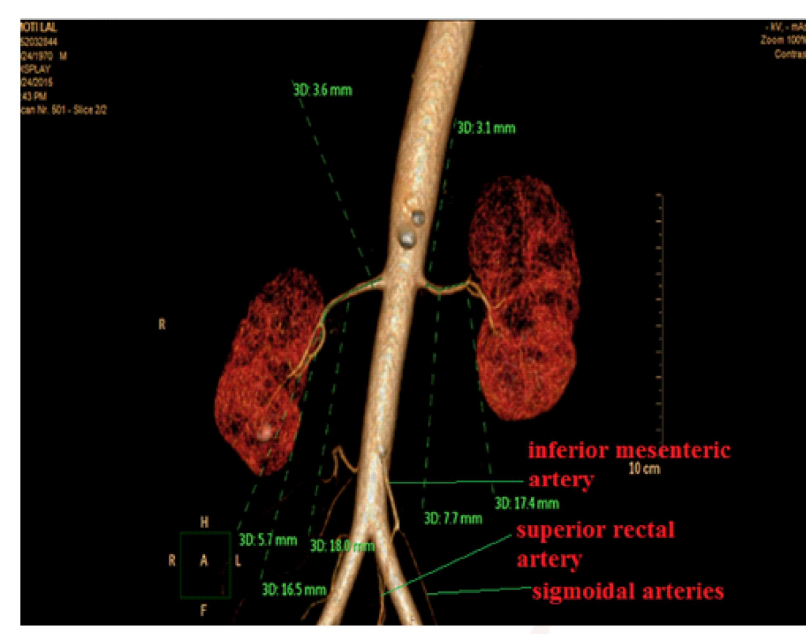

Fig. 2: Type I I-Absence of Left Colic Artery (6\%)

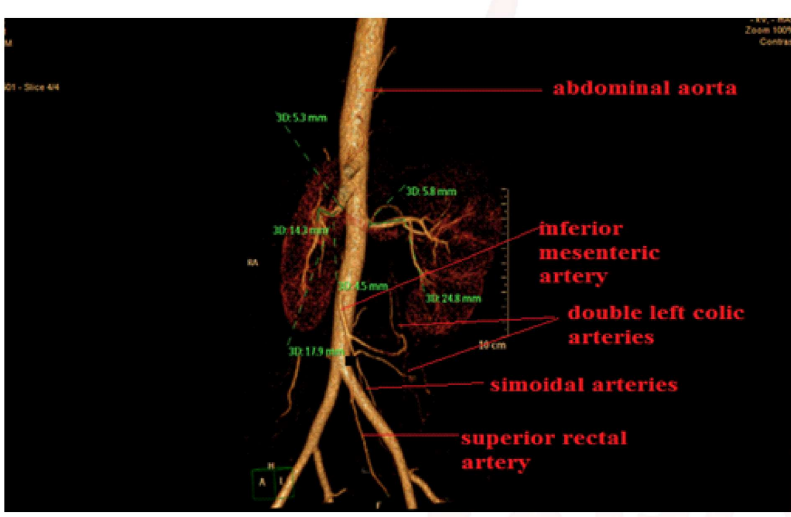

Fig. 3: Type III-Presence of Double Left Colic Artery (3\%)

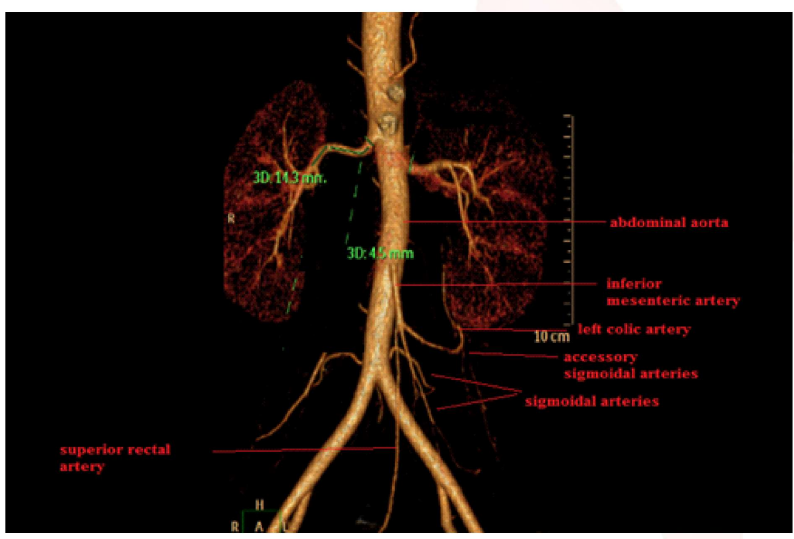

Fig. 4: Type IV -Presence of Accessory Sigmoidal Arteries (4\%).

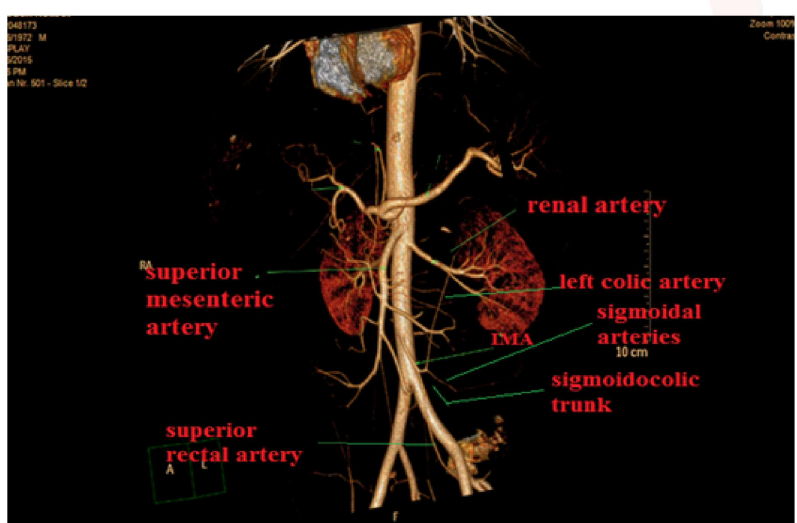

Fig. 5: Type V-Sigmoido Colic- Trunk (Ima-Inferior Mesenteric Artery)(4\%).

\section{DISCUSSION}

Aberrations in the emergence of the gut vessels are because of deviation from normal embryological development. From dorsal aspect of arteria maxima lying in abdomen, seven splanchnic vessels arises which supply the yolk sac (Chevallier, 1998). An anastomosis formed by these vessels in front of arteria maxima. During development, a huge amount of interconnections are vanished to form the normal vasculature of gut and this results in formation of three main trunks- (Coeliac, Superior mesenteric and Inferior mesenteric arteries), these further furcated to from segmental branches. The first three segments combine to originate as the coeliac trunk, the fourth segment originates as the Superior mesenteric artery, and the seventh segment originates as the inferior mesenteric artery.

According to Rekha Mane et. al. (2015) [5] mean length of inferior mesenteric artery is $3.25 \mathrm{~cm}$. According to this, inferior mesenteric artery gave three branches Left colic artery (98\%), sigmoidal and superior rectal artery (100\%). In $2 \%$ of cases Left colic artery was absent.

In thepresent study, 83\% cases in angiography showing normal branching pattern, $17 \%$ (angiography) cases showing variant anatomy as shown in Table-1.

Lee McGregor et al [6] (1995) observed that Left colic artery was absent in $6 \%$ of cases, variations also observed in thepresent study, $7.5 \%$ showing absence of left colic artery.

In the present study, we found common sigmoido-colic trunk which divides in to sigmoidal and left colic arteries. It was in $4 \%$ cases in angiographical data and but according to Nelson et al [7] (1988) prevalence of this variation is higher.

According to present study $4 \%$ cases observed with accessory sigmoidal arteries arise from left colic artery.

In present study according to angiographical method $3 \%$ cases are observed with double left colic arteries. These are very rare variation which is not detailed in previous studies however present study highlights these variations. These are very rare variation which is not detailed in 
previous studies however present study highlights these variations. The present study highlights that angiographic method isvery helpful for find the variations of vessels. Preoperative knowledge of such variations on the part of surgical team is exceedingly important to avoid arterial injury, predominantly in light of the increasing number of laparoscopic interventions.

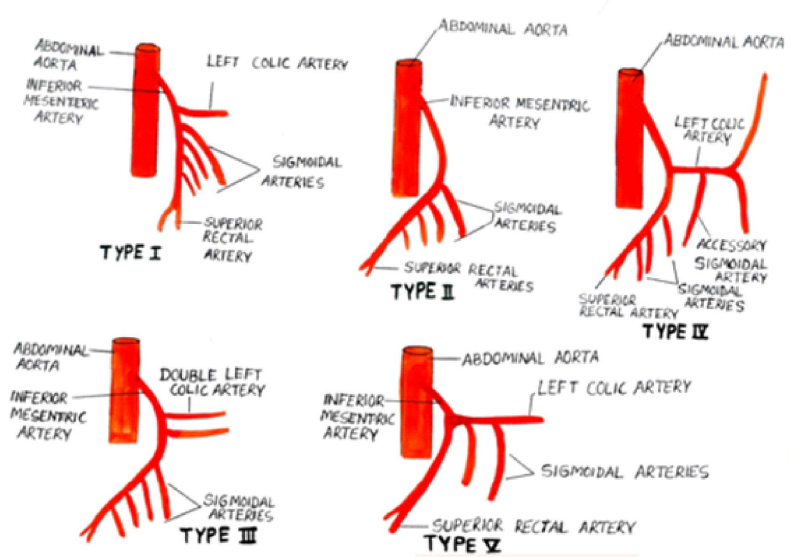

Fig. 6:Diagrammatic representation of branching pattern of inferior mesenteric artery.

\section{CONCLUSION}

To conclude, the inferior mesenteric artery which is the artery of the hindgut is known for its variation. The variations were seen in the branching pattern, according to these variations we divided branching pattern of artery in various types. These variations should be kept in mind during surgical and radiological procedures in abdomen to avoid intraoperative and post-operative complications.

\section{ACKNOWLEDGEMENTS}

We would like to sincerely thank Department of Anatomy of S.M.S.Medical College for allowing us to do our research.Dr. DhirajSaxena sir, who provided expertise that greatly, helped us to finish our research.

\section{Conflicts of Interests: None}

\section{REFERENCES}

[1]. Standring S, editor. Gray's Anatomy e-Book: 40 thEd, The Anatomical Basis of Clinical Practice. Elsevier Health Sciences; 2015 Aug 7.

[2]. Narendra Pamidi, Sreedher Varma And Venkata RamanaVollala. Anomalous branching pattern of coeliac trunk. International Journal of Anatomical Variations 2008;1:8-9.

[3]. Çiçekcibaoi $A E$, Ziylan $T$, Salbacak $A$, a eker $M$, Büyükmumcu $M$, Tuncer I. An investigation of the origin, location and variations of the renal arteries in human fetuses and their clinical relevance. Annals of Anatomy-AnatomischerAnzeiger. 2005 Sep 1;187(4):421-427. https://doi.org/10.1016/j.aanat.2005.04.011 PMid:16163857

[4]. Rubin GD, Alfrey EJ, Dake MD, Semba CP, Sommer FG, Kuo PC, Dafoe DC, Waskerwitz JA, Bloch DA, Jeffrey RB. Assessment of living renal donors with spiral CT. Radiology. 1995 May;195(2):457-462. https://doi.org/10.1148/radiology. 195.2.7724766 PMid:7724766

[5]. Mane RM, Shinde C. A Morphometric study of inferior mesenteric artery and its branches. International Journal of Medical Research and Review. 2015 May 31; 3(04):372-377.

https://doi.org/10.17511/ijmrr.2015.i4.070

[6]. Decker GAG, du Plesis DJ. Lee McGregor's: A Synopsis of Surgical Anatomy.12th ed. Bombay, India: Varghese Publishing House; 1995. P.226-228.

[7]. Nelson TM, Pollak R, Jonasson O, Abcarian H. Anatomic variants of the celiac, superior mesenteric, and inferior mesenteric arteries and their clinical relevance. Clinical Anatomy. 1988; 1(2):75-91. https://doi.org/10.1002/ca.980010202

How to cite this article:

Bali Sharma, Neha Saini, Dhiraj Saxena. ANOMALOUS BRANCHING PATTERN OF ARTERY OF HIND GUT-CT ANGIOGRAPHY BASED STUDY. Int J Anat Res 2020;8(3.1):76177620. DOI: $10.16965 /$ ijar.2020.174 